\title{
Retrotransposons Represent the Most Labile Fraction for Genomic Rearrangements in Polyploid Plant Species
}

\author{
M. Bento D. Tomás W. Viegas M. Silva \\ Centro de Botânica Aplicada à Agricultura, Secção de Genética, Instituto Superior de Agronomia, \\ Universidade Técnica de Lisboa, Lisboa, Portugal
}

\author{
Key Words \\ Arabidopsis · Genomic rearrangements · Polyploidy . \\ Retrotransposons - Triticeae
}

\begin{abstract}
Understanding how increased genome size and diversity within polyploid genomes impacts plant evolution and breeding continues to be challenging. Although historical studies by McClintock suggested the importance of transposable elements mediated by polyploidisation on genomic changes, data from plant crosses remain scarce. Despite the absence of a conclusive proof regarding autonomous retrotransposon movement in synthetic allopolyploids, the transposition of retrotransposons and their ubiquitous dispersion in all plant species might explain the positive correlation between the genome size of plants and the prevalence of retrotransposons. Here, we address polyploidisationmediated rearrangements of retrotransposon-associated sequences and discuss a tendency for a preferential restructuring of large ancestral genomes after polyploidisation. A comparative analysis of the frequency of modifications of retrotransposon-associated sequences in synthetic polyploids with marked differences in genome sizes is presented. Such analyses suggest the absence of a significant difference in the rates of rearrangements despite vast dissimilarities in the retrotransposon copy number between species, which
\end{abstract}

\section{KARGER}

E-Mail karger@karger.com

www.karger.com/cgr emphasises the high plasticity of this genomic feature. See also the sister article focusing on animals by Arkhipova and Rodriguez in this themed issue. Copyright $\odot 2013 \mathrm{~S}$. Karger AG, Basel

Polyploidy results from the union of 2 or more genomes in the same nucleus and is the major mode of plant genome evolution that can be artificially recreated. In Angiospermae, $30-70 \%$ of plant species have a polyploid origin, and almost all present species are considered to be paleopolyploids [Wendel, 2000; Wolfe, 2001].

Polyploids are classified into auto- or allopolyploids according to their genomic origin and can exhibit different ploidy levels. Autopolyploids result from genome doubling within the same species, and allopolyploids are formed by the combination of 2 or more distinct but usually related genomes. A survey of the published literature revealed that autopolyploid formation is higher than that of allopolyploids, suggesting that autopolyploids are much more common than expected [Ramsey and Schemske, 1998], though the consequences of these evolutionary outcomes are still elusive [Parisod et al., 2010b]. Two prevailing models that can explain the natural emergence of allopolyploids are the '2-step' model, involving interspecific hybridisation followed by chromosome doubling of the F1 hybrid, and the 'one-step' model, which is based 
on fertilisation of unreduced gametes from different diploid species or direct interspecific hybridisation between distinct autotetraploid species [Chen and Ni, 2006]. Autopolyploids can arise by either model involving only one species; however, it is usually believed that most autoplolyploids resulted from fertilisation of unreduced gametes because spontaneous chromosome doubling is a rare event in nature [Chen and $\mathrm{Ni}, 2006$ ].

As first suggested by McClintock [1984], the rise of both auto- and allopolyploids can induce a 'genomic shock', which is responsible for several genomic modifications that seem to be more pronounced in allopolyploids, reflecting their basic genome incompatibilities. The genetic and epigenetic modifications involved in the reorganisation of allopolyploid paternal genomes are extensively documented [Comai, 2000; Ma and Gustafson, 2005; Chen et al., 2008; Feldman and Levy, 2009; Jones and Hegarty, 2009], reaching from the number or organisation of chromosomes to genomic changes associated with genome downsizing and sequence modifications. In 1984, McClintock suggested that such widespread genomic changes can result from a higher activity of transposable elements (TEs). TEs constitute one of the main types of repetitive sequences, representing stretches of DNA that move throughout the genome and typically fall into 2 basic classes based on their transposition intermediate: RNA (retrotransposons or class I) and DNA (DNA transposons or class II). DNA transposons are present at low or moderate frequency in almost all eukaryotes, moving via DNA intermediates by either a 'cut-and-paste' mechanism or through DNA replication [Wicker et al., 2007]. Retrotransposons transpose via RNA intermediates without excising the original copy, are ubiquitously dispersed in all plant species and represent more than $50 \%$ of the genomic sequence in some cases [Kumar and Bennetzen, 1999; Lisch, 2013]. The retrotransposon 'copy-and-paste' mechanism of transposition introduces new copies of the original retroelement into the genome and may rapidly increase the frequency of repetitive elements in plant genomes [Schulman et al., 2004; Dvořák, 2009]. Such a tendency for an increase in retrotransposon copy number is counteracted by sequence deletions such as illegitimate retrotransposon recombination, which results in retrotransposon excision [Devos et al., 2002]. Thus, the ratio between TE insertions and deletions modulates the amount of repeated DNA in a genome and ultimately is largely responsible for the size of the genome [Dvořák, 2009].

Plant retrotransposon dynamics seems moreover to be influenced by different environmental conditions be- cause it was demonstrated that various biotic and abiotic stresses, such as infection, cold, heat, hybridization, or generation of doubled haploids, increase their transcriptional activity [Bennetzen, 2000; Mansour, 2007]. Retrotransposons can, therefore, be a source of innumerous mutations, deletions, insertions, frameshifts, inversions, translocations, and duplications that play major roles in structural genomic changes [Kumar and Bennetzen, 1999; Lippman et al., 2004] and may affect gene functions through cis and/or trans gene regulation [Chen and $\mathrm{Ni}$, 2006]. Although many studies review the importance of polyploidy or retrotransposons in plants, most consider them as separate issues, and few discuss the impact of polyploidisation on transposable element dynamics. In this article, we review the influence of polyploidisation on the retrotransposon copy number and genomic rearrangements associated with retrotransposon elements. The role of retrotransposons in genomic alterations in species with large and small genomes is further discussed based on new results from Arabidopsis allopolyploids.

\section{Retrotransposon Classes and Abundance in Plant Genomes}

Retrotransposons classify into 2 major categories: long terminal repeats (LTR) containing elements (LTR retrotransposons, the most numerous class of large retroelements) and non-LTR retrotransposons (fig. 1). LTR retrotransposons are further divided into Copia and Gypsy groups according to the degree of sequence similarity and coding sequence organisation. Both Copia and Gypsy retrotransposons are present throughout the plant kingdom and are normally found in high copy number - up to a few million copies in plants with large genomes [Kumar and Bennetzen, 1999]. LTR retrotransposons differ greatly in size, ranging from a few hundred base pairs to very large LTR retrotransposons, such as the 25-kb element Ogre in peas [Neumann et al., 2003]. The sequences flanking LTRs also vary from several hundred base pairs to more than $5 \mathrm{~kb}$ [Wicker et al., 2007] and do not encode any known proteins but contain promoters and terminators that regulate their transcriptional activity [Kumar and Bennetzen, 1999]. Large retrotransposon derivate elements with lengths exceeding $4 \mathrm{~kb}$ and terminal repeat retrotransposons in miniature with lengths smaller than $4 \mathrm{~kb}$ also belong to the LTR retrotransposon class but lack coding sequences which qualifies them as nonautonomous elements. Illegitimate LTR recombination results in soloLTRs originating from retrotransposon excision and may 
Fig. 1. Class I transposable elements or retrotransposons observed in plant genomes. Retrotransposons can be flanked by long terminal repeats (LTRs) and are further classified into Copia and Gypsy classes according to the architecture of their coding sequence organisation, composed of capsid protein (GAG), protease (PR), integrase (INT), reverse transcriptase (RevT), and RNase $\mathrm{H}$ (RNASE H) genes. Terminal-repeat retrotransposons in miniature (TRIMs) and large retrotransposon derivates (LARDs) lack coding sequences and, thus, are nonautonomous. Non-LTR retrotransposons are classified into short interspersed nuclear elements (SINEs), which contain a Pol III promoter and a $3^{\prime}$ variable region, and long interspersed nuclear elements (LINEs), which encode integrase, endonuclease, reverse transcriptase, and a variable region. $\mathrm{EN}=$ endonuclease; $(\mathrm{N})_{\mathrm{n}}=$ variable region. Adapted from [Parisod et al., 2010a].

\section{Class I transposable elements or retrotransposons}

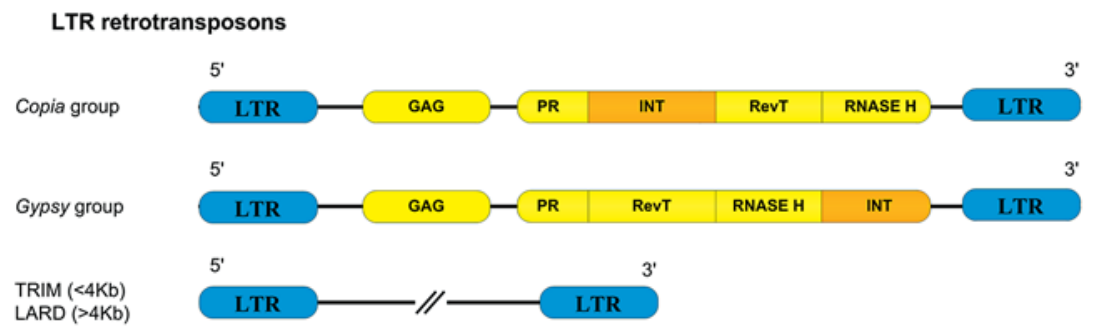

Non-LTR retrotransposons

SINE

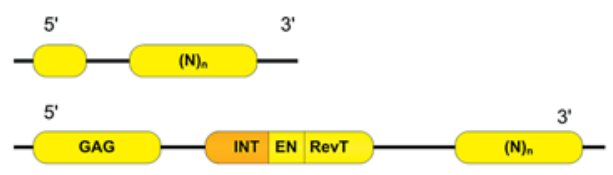

also occur between neighbouring retrotransposons of the same type [Devos et al., 2002]. Non-LTR retrotransposons are classified into short interspersed nuclear elements (SINE), which encompass $80-500 \mathrm{bp}$ and are relatively rare in most plant genomes, and long interspersed nuclear elements (LINE), which can comprise several kilobases in length, possibly representing the most ancient class of retroelements [Wicker et al., 2007]. In fact, several studies suggest that initial LTR retrotransposons may have resulted from the incorporation of LTRs into LINEs [Bennetzen, 2000].

An extensive study of the Triticeae tribe, which encompasses species with large genomes, revealed that TEs represent up to $80 \%$ of those genomes and are comprised of retrotransposons, of which a larger fraction are Gypsylike (corresponding approximately to $70 \%$ of the characterised TEs) and Copia-like retrotransposons (representing $\sim 20 \%$ ) as well as other less frequent classes [Middleton et al., 2013]. A positive correlation between plant genome size and retrotransposon frequency can easily be observed considering that in species with large genomes, such as Triticum aestivum (17,000 Mb), Aegilops tauschii (4,000 $\mathrm{Mb})$ and Zea mays (2,665 Mb), retrotransposons represent $\sim 63, \sim 53$, and $\sim 49 \%$ of these genomes, respectively [Bennett and Smith, 1976; Arumuganathan and Earle, 1991; Meyers et al., 2001; Li et al., 2004; Brenchley et al., 2012]. In contrast, in species with smaller genomes, such as Brassica oleracea (758 Mb), Oryza sativa (489 Mb) or Arabidopsis thaliana (125 Mb), retrotransposon elements

Retrotransposon Rearrangements in

Polyploid Plant Species only represent $\sim 14, \sim 12$ and $\sim 5.6 \%$ of these genomes [Bennett and Smith, 1976; Olszewska and Osiecka, 1984; Arabidopsis Genome Initiative, 2000; Mao et al., 2000; Peterson-Burch et al., 2004; Zhang and Wessler, 2004].

It is plausible to believe that distinct retrotransposon families have descended from few ancient evolutionary lineages because a common origin was found for Copialike retrotransposons present in wheat, rice and Arabidopsis [Wicker and Keller, 2007]. In addition to the existence of thousands of different retrotransposon families, it is quite interesting to note the dearth of families specific to only one species [Wicker and Keller, 2007] because almost all seem to be present in most genomes at least at a very low frequency [Middleton et al., 2013]. Through an in-depth study of 10 Triticeae taxa, Middleton et al. [2013] demonstrated that in each species, few families are very abundant and represent a large fraction of repetitive elements in large genomes. Similar disparity in the copy number of distinct LTR families was additionally observed in B. rapa [Wang et al., 2011]. Large sequencing projects in wheat additionally revealed that most LTR elements described are truncated [Choulet et al., 2010; Brenchley et al., 2012], and most likely originated from internal deletions, nested insertions and illegitimate recombination events resulting from the coexistence of TE families in certain chromosomal domains. Both in Triticeae species as well as in maize, most intergenic regions enriched in LTR retroelements are organised in complex nested insertions [SanMiguel et al., 1996; 
Dvořák, 2009]. Such nested arrangements of retrotransposons are rarely observed in Arabidopsis and Brassica species, which is in clear contrast to evidence from Triticeae [Arabidopsis Genome Initiative, 2000; PetersonBurch et al., 2004; Alix et al., 2005; Wang et al., 2011].

\section{Retrotransposons Are Involved in Polyploidisation- Associated Genomic Alterations}

Autopolyploids are classified into 2 main types: 'typical' autopolyploids, characterised by multivalent pairing at meiosis and multisomic inheritance, and 'cytologically diploidised' autopolyploids, which exhibit almost exclusively homologous pairing at meiosis, although they have more than 2 genome copies [Eilam et al., 2010]. Cytologically diploidised autotetraploids display considerable genome downsizing immediately after autopolyploidisation, such as in the synthetic autopolyploid Phlox drummondii, where reductions of up to $\sim 25 \%$ of the total parental DNA content in the third generation have been observed [Eilam et al., 2010]. In contrast, typical autopolyploids usually exhibit additive genome size values [Eilam et al., 2010]. Genomic studies of autopolyploids have been restricted to AFLP (amplified fragment length polymorphism) analyses, such as in Arabidopsis, where no significant changes were detected after autopolyploidisation [Ozkan et al., 2006]. The involvement of retrotransposons in autopolyploid genomic alterations has yet to be addressed, although activation of the En/Spmlike DNA transposon (Sunfish) was reported after investigating transposon instability by microarray analysis (and confirmed by a methyl-insensitive Southern blot) in A. thaliana autotetraploids [Madlung et al., 2005].

The impact of allopolyploidisation on transposable elements has been studied in few polyploid species, as reviewed by Parisod et al. [2010a], and the information regarding its influence on retrotransposons is even sparser. Despite reported changes in retrotransposon transcription, there is still a lack of evidence regarding autonomous retrotransposable element movement in synthetic allopolyploids [Kashkush et al., 2003]. For instance, in the allopolyploid Aegilops sharonensis $\times$ T. monococcum, the analysis of novel bands observed by cDNA-AFLP revealed the synthesis of new transcripts from adjacent sequences of Wis2-1A retrotransposon including antisense or sense genes, although no evidence of polyploidy-induced transposition was detected [Kashkush et al., 2003]. Although there is a lack of information regarding the effects of allopolyploidisation on retrotransposons, a study of the nonautonomous terminal repeat retrotransposons in miniature family $V e j u$ in allohexaploid wheat revealed a massive elimination (50\%) of Veju LTRs in the first generation, followed by a burst in subsequent generations, which led to a marked increase in the Veju element copy number [Kraitshtein et al., 2010]. Transposition events were also reported for class II elements in allohexaploid wheat species [Yaakov et al., 2013]. Dot-blot analysis of natural wheat also indicated transposition bursts because the observed retrotransposon copy number was higher than the expected addition of retrotransposons in parental species. However, using the same methodology, no differences were detected in retrotransposon copy number immediately after polyploidisation in synthetic allopolyploids $A$. speltoides $\times T$. urartu, A. sharonensis $\times T$. monococcum, $A$. speltoides $\times A$. tauschii, and T. turgidum $\times A$. tauschii when compared with parental lines, suggesting that copy number variation occurs progressively after polyploidisation [Li et al., 2004].

IRAP (interretrotransposon amplified polymorphism) and REMAP (retrotransposon microsatellite amplified polymorphism) have also been important tools to evaluate the involvement of retrotransposon elements in allopolyploid genomic rearrangements. Using such markers in a comparative genomic analysis of the synthetic allopolyploid triticale (T. aestivum $\times$ Secale cereale) and the parental genomes, it was demonstrated that $\sim 28 \%$ of retrotransposons and microsatellite-related sequences are rearranged in the polyploid [Bento et al., 2008]. Most rearrangements resulted from losses of parental bands, affecting both repetitive and coding sequences, and a minor frequency $(4.2 \%)$ corresponded to novel bands. In the natural allopolyploid Spartina anglica, evidence of major changes in $\mathrm{CpG}$ methylation in the proximity of retrotransposon insertions were found by sequence-specific amplified polymorphism (SSAP) analysis; however, few new TE insertions were proposed among the retrotransposon families investigated (terminal-repeat retrotransposons in miniature, Cassandra and Wis Copia-like retrotransposons) [Parisod et al., 2009], although some fragment losses were detected immediately after hybridisation [Parisod et al., 2010a]. More recently, a study of Athilalike retrotransposons by SSAP in the synthetic allotetraploids $B$. napus revealed mostly additive profiles when compared with the diploid parents, and the characterisation of nonadditive SSAP bands indicated that genomic rearrangements had occurred rather than new transposition events [Sarilar et al., 2013]. Similar analyses revealed a proliferation of the Tnt 1 retrotransposon after genome doubling in the synthetic Nicotiana tabacum. Structural 


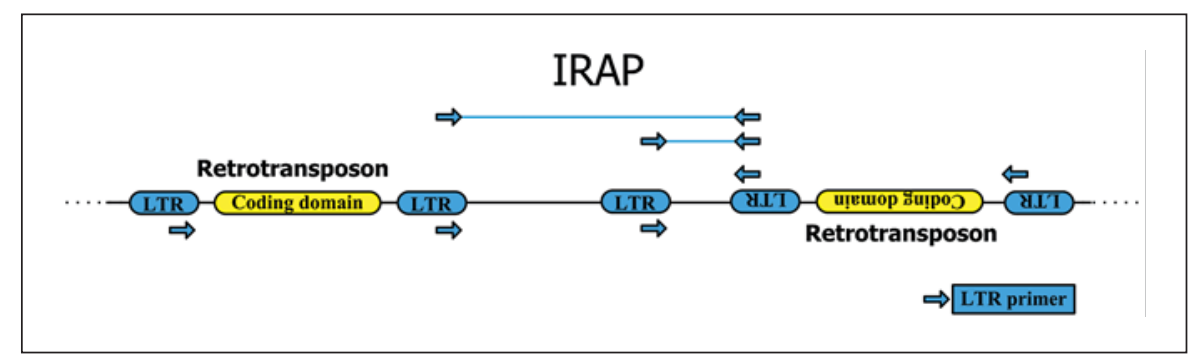

Fig. 2. Principles of IRAP (interretrotransposon amplified polymorphism). LTR primers (blue arrows) facing outward from the ends of LTRs will amplify intervening DNA from retrotransposons or solo-LTRs in opposite orientations. Retrotransposons or soloLTRs in the same orientation will not result in amplification prod- ucts. Adapted from Kalendar and Schulman [2006]. IRAP analysis allowed the characterisation of Gypsy-like Athila4-6 and Tat1 retrotransposon adjacent sequences (see online suppl. table 1, www. karger.com/doi/10.1159/000353308) in Arabidopsis polyploid lines. changes affecting retrotransposons and adjacent sequences were also predominantly represented by losses of SSAP fragments of paternal origin, including indels or the complete loss of Tnt 1 elements [Petit et al., 2010]. In contrast, in the natural allopolyploid S. anglica, losses of SSAP fragments from maternal origin seemed to predominate [Parisod et al., 2009]. Progenitor biases for rearrangements through IRAP and REMAP analyses were also observed in triticale, where the paternal rye genome is considerably more reorganised than the wheat genome [Bento et al., 2008]. A further in-depth analysis of Triticeae polyploids additionally revealed that the larger genome (comparing DNA content per haploid genome) is usually more affected independently of its maternal or paternal status [Bento et al., 2011]. Jiang et al. [2011] also corroborated this suggestion through an IRAP and REMAP study of the neosynthesised Cucumis allotetraploid, which revealed that $18 \%$ of the rearranged bands corresponded mainly to a loss from the larger parental genome.

Although it was previously demonstrated that the parental genome size is correlated with the frequency of rearrangements in its allopolyploid, no comparative analysis of genomic changes among allopolyploids with very different genome sizes has been performed. Genome sizes can vary as much as 1,000-fold between plant species from the small genome of $A$. thaliana, which is approximately $125 \mathrm{Mb}$ [Arabidopsis Genome Initiative, 2000], to species with very large genomes, such as T. aestivum (17 Gb) [Brenchley et al., 2012] or Fritillaria assyriaca (123 Gb) [Bennett and Smith, 1976]; therefore, the relevance of genome size on the frequencies of polyploidisationinduced retrotransposon-related sequence rearrangements remains to be understood.

Retrotransposon Rearrangements in Polyploid Plant Species

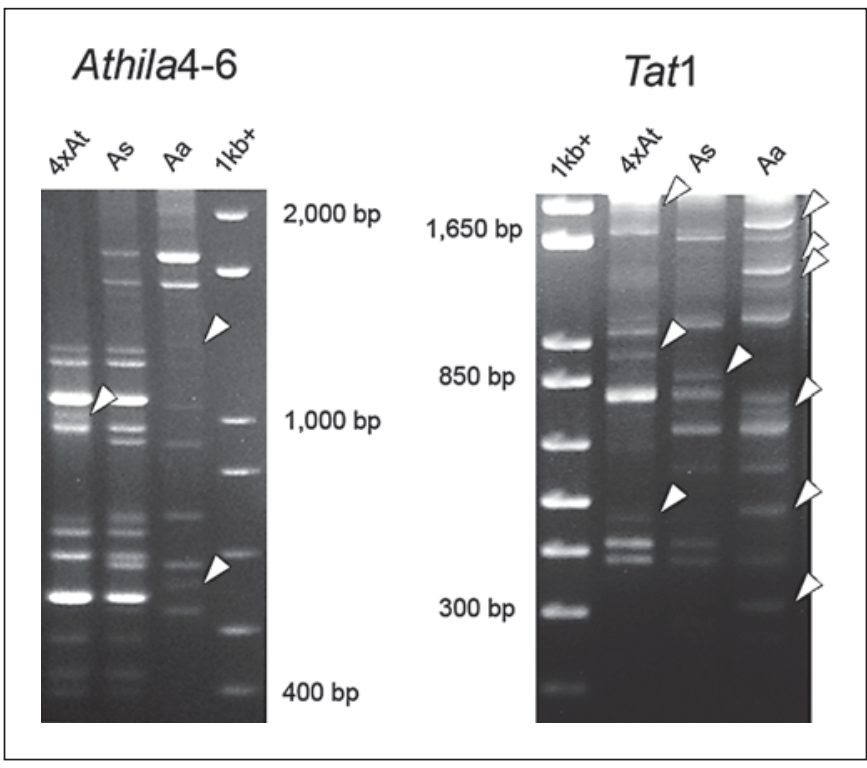

Fig. 3. IRAP banding profiles obtained with primers for Athila4-6 and Tat 1 of $A$. thaliana LC612 $2 \mathrm{n}=4 \times=20(4 \times \mathrm{At})$, A. suecica synthetic allopolyploid $2 n=4 \times=26$ (As) and A. arenosa CARE-1 $2 n=4 \times=32(\mathrm{Aa})$. Arrowheads indicate rearranged bands.

\section{Frequency of Retrotransposon Rearrangements Is Independent of Allopolyploid Genome Sizes}

Because the contribution of retrotransposons to rearrangements in allopolyploids with small genomes had not yet been evaluated, we used the IRAP methodology (fig. 2) to study a newly synthesised $A$. suecica line $(2 n=4 \times=26$, F4 generation, NASC stock number N3899) produced by L. Comai's laboratory [Madlung et al., 2005]. The results obtained through a comparison of the profiles (fig. 3) of 
Table 1. IRAP analyses of the synthetic allopolyploid A. suecica and its parental lines

\begin{tabular}{|c|c|c|c|}
\hline & \multicolumn{2}{|l|}{ IRAP } & \multirow[t]{2}{*}{ Total } \\
\hline & Athila4-6 & Tat 1 & \\
\hline A. thaliana tetraploid & 12 & 8 & 20 \\
\hline A. arenosa & 8 & 12 & 20 \\
\hline \multicolumn{4}{|l|}{ A. thaliana vs. A. arenosa } \\
\hline Monomorphic bands ${ }^{\mathrm{a}}$ & 0 & 2 & 2 \\
\hline Polymorphic bands ${ }^{\mathrm{b}}$ & 20 & 16 & 36 \\
\hline \multicolumn{4}{|l|}{ A. suecica } \\
\hline Expected $^{\mathrm{c}}$ & 20 & 18 & 38 \\
\hline Observed bands ${ }^{\mathrm{d}}$ & 17 & 10 & 27 \\
\hline Rearranged bands ${ }^{\mathrm{e}}$ & 3 & 10 & 13 \\
\hline Lost from $A$. thaliana & 1 & 3 & 4 \\
\hline Lost from $A$. arenosa & 2 & 6 & 8 \\
\hline Novel bands & 0 & 1 & 1 \\
\hline
\end{tabular}

${ }^{\mathrm{a}}$ Common to both $A$. suecica progenitors; ${ }^{\mathrm{b}}$ observed in only one A. suecica progenitor; ${ }^{c}$ sum of parental bands observed; ${ }^{d}$ bands observed in the allopolyploid; ${ }^{\text {e parental }}$ bands absent and novel bands only observed in the polyploid.
Table 2. Comparative analysis of AFLP and IRAP results in A. suecica and octoploid triticale (Triticum aestivum 'Chinese Spring' $\times$ Secale cereale 'Imperial') allopolyploids

\begin{tabular}{|c|c|c|c|c|}
\hline & \multicolumn{2}{|l|}{ AFLP } & \multicolumn{2}{|l|}{ IRAP } \\
\hline & bands affected, n & total $^{\mathrm{a}}$ & bands affected, n & total $^{\mathrm{a}}$ \\
\hline A. suecica & $7(2.3 \%)$ & $308^{\mathrm{b}}$ & $13(33.3 \%)$ & $39^{d}$ \\
\hline Triticale & $830(43.4 \%)$ & $1,910^{c}$ & $15(27.8 \%)$ & $54^{\mathrm{e}}$ \\
\hline
\end{tabular}

a Total number of bands = expected bands corresponding to the sum of parental bands and allopolyploid novel bands not observed in any parental profile; ${ }^{b}$ Madlung et al., 2005; ${ }^{\mathrm{c}} \mathrm{Ma}$ et al., 2004; ${ }^{\mathrm{d}}$ in the present paper; ${ }^{\mathrm{e}}$ Bento et al., 2008.

Note: The $\chi^{2}$ test was used to compare the results obtained for different species with the same technique and with different techniques for the same species. the exact parental lines A. thaliana autotetraploid (LC612 line, $2 \mathrm{n}=4 \times=20$, NASC stock number N3900) and $A$. arenosa (CARE- 1 line, $2 \mathrm{n}=4 \times=32$, NASC stock number N3901) were consistently reproduced in all PCR replicates from at least 3 distinct plants of each genotype (summarised in table 1). A high frequency (33\%) of rearrangements ( 13 rearranged/39 total bands) was detected in the synthetic allopolyploid $A$. suecica comprising $92 \%$ parental band losses and only one novel band.

The frequency of allopolyploidisation-induced retrotransposon-associated genomic changes was further compared between A. suecica and triticale [Bento et al., 2008], based on IRAP analyses (table 2). Interestingly, regarding the large differences in retrotransposon copy number between these 2 allopolyploid model species, no significant difference $(\mathrm{p}=0.62)$ was detected in the number of bands affected (33\% in A. suecica and $28 \%$ in triticale). Thus, it is clear that retrotransposons represent a very labile portion of the genome, which is highly affected by polyploidisation yet is apparently independent of genome sizes. Such retrotransposon-associated genomic modifications are enigmatic; however, when comparing genomic rearrangement frequencies evaluated through AFLP, significant differences $\left(\mathrm{p}=6.58 \times 10^{-40}\right)$ between both polyploids are detected; specifically, $43 \%$ of restructured bands were detected in triticale, and only $2.3 \%$ were detected in A. suecica [Ma et al., 2004; Madlung et al., 2005]. When comparing IRAP and AFLP, a significant difference in the frequency of rearrangements was observed in A. suecica $\left(\mathrm{p}=2.55 \times 10^{-12}\right)$, but not in triticale $(\mathrm{p}=0.14)$. 


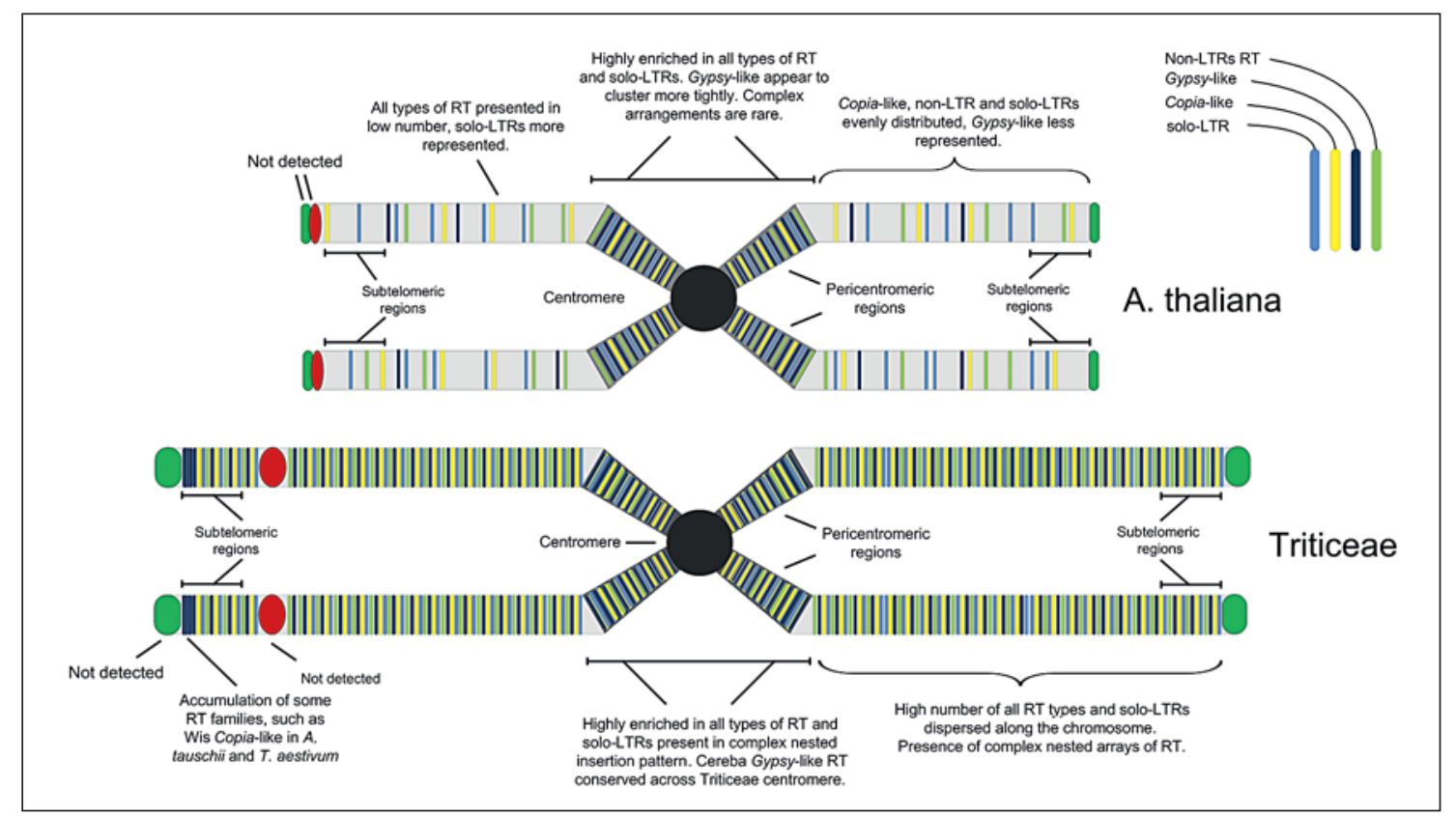

Fig. 4. Schematic representation of the distribution of retrotransposons (RT) in plant chromosomes. The upper part represents $A$. thaliana, and the lower part represents Triticeae species (telomeres in green, nucleolus organiser regions [NORs] in red). In A. thaliana, retrotransposons tend to be clustered within pericentromeric heterochromatin and are less represented across chromosome arms; Gypsy-like elements are especially abundant in pericentro- meric regions. The presence of complex nested arrays of retrotransposon is rare. In Triticeae, retrotransposons may be clustered in heterochromatin domains but are also present in high copy number all over chromosome arms, frequently in complex nested insertions. Chromosomes are not drawn to scale [Li et al., 2004; Peterson-Burch et al., 2004; Dvořák, 2009].
Considering that AFLP detects changes in restriction fragments from random loci allowing analyses across the genome [Madlung et al., 2005], whereas IRAP assesses particularly labile repetitive sequences, such as retrotransposons [Kalendar and Schulman, 2006], our preliminary results suggest that domains rich in retrotransposon-related sequences are predominantly affected in A. suecica, whereas rearrangements in triticale appear to occur throughout the genome.

A plausible explanation for the discrepant rearrangement frequencies detected through AFLP and IRAP in triticale and A. suecica can be found by examining the differential retrotransposon chromosomal distribution in both species. The results of retrotransposon mapping through in situ hybridisation, which were later supported by largescale sequencing initiatives, suggest that the retrotransposon distribution patterns clearly differ between species with large and small genomes (reviewed in fig. 4). The study of the chromosomal distribution of retrotransposons in T. aestivum through FISH (fluorescent in situ hybridisation) revealed that most Gypsy-like retrotransposon elements analysed, such as Sabrina, Wham, Wilma, Nusif, and Fatima as well as Copia-like retrotransposons Angela and Wis, are detected over the entire length of all chromosomes [Li et al., 2004]. Choulet et al. [2010] also confirmed this highly widespread distribution of retrotransposons in T. aestivum. In the small genome of Arabidopsis, most copies of Gypsy-like, Copia-like and non-LTR retrotransposons are preferentially clustered in pericentromeric heterochromatin. Nevertheless, Copia-like and non-LTR retrotransposons differ in their genomic organisation from Gypsy-like retrotransposons and are more loosely associated with pericentromeric regions, being widespread throughout chromosomes though in low copy number [Peterson-Burch et al., 2004]. A similar distribution of retrotransposons was also observed in closely related species such as B. oleracea and B. rapa, where most retroelements clustered in pericentromeric heterochromatin, yet coincided with a wide distribution of Athila-like Gypsy elements throughout chromosomes [Alix et al., 2005; Wang et al., 2011]. Thus, our comparative analysis suggests that retrotransposon elements might be similarly rearranged in 
model allopolyploid species with both large and small genomes, independent of their distinct abundance and distribution patterns. However, the understanding of the real causes for the discrepancy between AFLP and IRAP in detecting rearrangement frequencies in triticale and A. suecica requires further research to extensively characterise the restructured bands at the sequence level.

\section{Acknowledgements}

M. Bento is funded by a postdoctoral grant (SFRH/BPD/ 80550/2011) by the Fundação para a Ciência e a Tecnologia (FCT), Portugal, and D. Tomás' Fellowship and the research work was financed by PTDC/BIA-BEC/101964/2008, FCT.

\section{References}

Alix K, Ryder CD, Moore J, King GJ, Pat HeslopHarrison JS: The genomic organization of retrotransposons in Brassica oleracea. Plant Mol Biol 59:839-851 (2005).

Arabidopsis Genome Initiative: Analysis of the genome sequence of the flowering plant Arabidopsis thaliana. Nature 408:796-815 (2000).

Arumuganathan K, Earle ED: Nuclear DNA content of some important plant species. Plant Mol Biol Rep 9:208-218 (1991).

Bennett MD, Smith JB: Nuclear DNA amounts in angiosperms. Philos Trans R Soc Lond B Biol Sci 274:227-274 (1976).

Bennetzen JL: Transposable element contributions to plant gene and genome evolution. Plant Mol Biol 42:251-269 (2000).

Bento M, Pereira S, Rocheta M, Gustafson P, Viegas W, Silva M: Polyploidization as a retraction force in plant genome evolution: sequence rearrangements in triticale. PLoS One 3:e1402 (2008).

Bento M, Gustafson JP, Viegas W, Silva M: Size matters in Triticeae polyploids: larger genomes have higher remodeling. Genome 54: 175-183 (2011).

Brenchley R, Spannagl M, Pfeifer M, Barker GL, D'Amore R, et al: Analysis of the bread wheat genome using whole-genome shotgun sequencing. Nature 491:705-710 (2012).

Chen ZJ, Ni ZF: Mechanisms of genomic rearrangements and gene expression changes in plant polyploids. Bioessays 28:240-252 (2006).

Chen M, Ha M, Lackey E, Wang JL, Chen ZJ: RNAi of met1 reduces DNA methylation and induces genome-specific changes in gene expression and centromeric small RNA accumulation in Arabidopsis allopolyploids. Genetics 178:1845-1858 (2008).

Choulet F, Wicker T, Rustenholz C, Paux E, Salse $J$, et al: Megabase level sequencing reveals contrasted organization and evolution patterns of the wheat gene and transposable element spaces. Plant Cell 22:1686-1701 (2010).

Comai L: Genetic and epigenetic interactions in allopolyploid plants. Plant Mol Biol 43:387399 (2000).

Devos KM, Brown JK, Bennetzen JL: Genome size reduction through illegitimate recombination counteracts genome expansion in Arabidopsis. Genome Res 12:1075-1079 (2002).
Dvořák J: Triticeae genome structure and evolution, in Feuillet C, Muehlbauer GJ (eds): Genetics and Genomics of the Triticeae, pp 685711 (Springer, New York 2009).

Eilam T, Anikster Y, Millet E, Manisterski J, Feldman M: Genome size in diploids, allopolyploids, and autopolyploids of mediterranean Triticeae. Journal of Botany 2010:1-12 (2010).

Feldman M, Levy AA: Genome evolution in allopolyploid wheat - a revolutionary reprogramming followed by gradual changes. J Genet Genomics 36:511-518 (2009).

Jiang B, Lou Q, Wu Z, Zhang W, Wang D, et al: Retrotransposon- and microsatellite sequence-associated genomic changes in early generations of a newly synthesized allotetraploid Cucumis $\times$ hytivus Chen \& Kirkbride. Plant Mol Biol 77:225-233 (2011).

Jones RN, Hegarty M: Order out of chaos in the hybrid plant nucleus. Cytogenet Genome Res 126:376-389 (2009).

Kalendar R, Schulman AH: IRAP and REMAP for retrotransposon-based genotyping and fingerprinting. Nat Protoc 1:2478-2484 (2006).

Kashkush K, Feldman M, Levy AA: Transcriptional activation of retrotransposons alters the expression of adjacent genes in wheat. Nat Genet 33:102-106 (2003).

Kraitshtein Z, Yaakov B, Khasdan V, Kashkush K: Genetic and epigenetic dynamics of a retrotransposon after allopolyploidization of wheat. Genetics 186:801-812 (2010).

Kumar A, Bennetzen JL: Plant retrotransposons. Annu Rev Genet 33:479-532 (1999).

Li W, Zhang P, Fellers JP, Friebe B, Gill BS: Sequence composition, organization, and evolution of the core Triticeae genome. Plant J 40: 500-511 (2004).

Lippman Z, Gendrel AV, Black M, Vaughn MW, Dedhia N, et al: Role of transposable elements in heterochromatin and epigenetic control. Nature 430:471-476 (2004).

Lisch D: How important are transposons for plant evolution? Nat Rev Genet 14:49-61 (2013).

Ma XF, Gustafson JP: Genome evolution of allopolyploids: a process of cytological and genetic diploidization. Cytogenet Genome Res 109: 236-249 (2005).

Ma XF, Fang P, Gustafson JP: Polyploidizationinduced genome variation in triticale. Genome 47:839-848 (2004).
Madlung A, Tyagi AP, Watson B, Jiang HM, Kagochi T, et al: Genomic changes in synthetic Arabidopsis polyploids. Plant J 41:221-230 (2005).

Mansour A: Epigenetic activation of genomic retrotransposons. J Cell Mol Biol 6:99-107 (2007).

Mao L, Wood TC, Yu Y, Budiman MA, Tomkins $\mathrm{J}$, et al: Rice transposable elements: a survey of 73,000 sequence-tagged-connectors. Genome Res 10:982-990 (2000).

McClintock B: The significance of responses of the genome to challenge. Science 226:792801 (1984).

Meyers BC, Tingey SV, Morgante M: Abundance, distribution, and transcriptional activity of repetitive elements in the maize genome. Genome Res 11:1660-1676 (2001).

Middleton CP, Stein N, Keller B, Kilian B, Wicker T: Comparative analysis of genome composition in Triticeae reveals strong variation in transposable element dynamics and nucleotide diversity. Plant J 73:347-356 (2013).

Neumann P, Pozárková D, Macas J: Highly abundant pea LTR retrotransposon Ogre is constitutively transcribed and partially spliced. Plant Mol Biol 53:399-410 (2003).

Olszewska MJ, Osiecka R: The relationship between 2 c DNA content, life-cycle type, systematic position and the dynamics of DNA endoreplication in parenchyma nuclei during growth and differentiation of roots in some dicotyledonous herbaceous species. Biochem Physiol Pfl 179:641-657 (1984).

Ozkan H, Tuna M, Galbraith DW: No DNA loss in autotetraploid of Arabidopsis thaliana. Plant Breeding 125:288-291 (2006).

Parisod C, Salmon A, Zerjal T, Tenaillon M, Grandbastien MA, Ainouche M: Rapid structural and epigenetic reorganization near transposable elements in hybrid and allopolyploid genomes in Spartina. New Phytol 184: 1003-1015 (2009).

Parisod C, Alix K, Just J, Petit M, Sarilar V, et al: Impact of transposable elements on the organization and function of allopolyploid genomes. New Phytol 186:37-45 (2010a).

Parisod C, Holderegger R, Brochmann C: Evolutionary consequences of autopolyploidy. New Phytol 186:5-17 (2010b). 
Peterson-Burch BD, Nettleton D, Voytas DF: Genomic neighborhoods for Arabidopsis retrotransposons: a role for targeted integration in the distribution of the Metaviridae. Genome Biol 5:R78 (2004).

Petit M, Guidat C, Daniel J, Denis E, Montoriol E, et al: Mobilization of retrotransposons in synthetic allotetraploid tobacco. New Phytol 186: 135-147 (2010).

Ramsey J, Schemske DW: Pathways, mechanisms, and rates of polyploid formation in flowering plants. Annu Rev Ecol Syst 29:467-501 (1998).

SanMiguel P, Tikhonov A, Jin YK, Motchoulskaia $\mathrm{N}$, Zakharov D, et al: Nested retrotransposons in the intergenic regions of the maize genome. Science 274:765-768 (1996).
Sarilar V, Palacios PM, Rousselet A, Ridel C, Falque $\mathrm{M}$, et al: Allopolyploidy has a moderate impact on restructuring at three contrasting transposable element insertion sites in resynthesized Brassica napus allotetraploids. New Phytol 198:593-604 (2013).

Schulman AH, Gupta PK, Varshney RK: Organization of microsatellites and retrostransposons in cereal genomes, in Gupta PK, Varshney RK (eds): Cereal Genomics, pp 83-118 (Kluwer Academic Publishers, Dordrecht 2004).

Wang $\mathrm{X}$, Wang $\mathrm{H}$, Wang J, Sun R, Wu J, et al: The genome of the mesopolyploid crop species Brassica rapa. Nat Genet 43:1035-1039 (2011).

Wendel JF: Genome evolution in polyploids. Plant Mol Biol 42:225-249 (2000).

Wicker T, Keller B: Genome-wide comparative analysis of copia retrotransposons in Triticeae, rice, and Arabidopsis reveals conserved ancient evolutionary lineages and distinct dynamics of individual copia families. Genome Res 17:1072-1081 (2007).
Wicker T, Sabot F, Hua-Van A, Bennetzen JL, Capy P, et al: A unified classification system for eukaryotic transposable elements. Nat Rev Genet 8:973-982 (2007)

Wolfe KH: Yesterday's polyploids and the mystery of diploidization. Nat Rev Genet 2:333341 (2001).

Yaakov B, Ben-David S, Kashkush K: Genomewide analysis of Stowaway-like MITEs in wheat reveals high sequence conservation, gene association, and genomic diversification. Plant Physiol 161:486-496 (2013).

Zhang X, Wessler SR: Genome-wide comparative analysis of the transposable elements in the related species Arabidopsis thaliana and Brassica oleracea. Proc Natl Acad Sci USA 101: 5589-5594 (2004). 